\title{
An active region jet observed with Hinode
}

\author{
C. Chifor ${ }^{1}$, P. R. Young ${ }^{2}$, H. Isobe ${ }^{3}$, H. E. Mason ${ }^{1}$, D. Tripathi ${ }^{1}$, H. Hara ${ }^{4}$, and T. Yokoyama ${ }^{3}$ \\ 1 Department of Applied Mathematics and Theoretical Physics, CMS, Wilberforce Road, Cambridge CB3 0WA, UK \\ e-mail: c.chifor@damtp.cam.ac.uk \\ 2 STFC Rutherford Appleton Laboratory, Chilton, Didcot, Oxfordshire OX11 0QX, UK \\ 3 Department of Earth and Planetary Science, University of Tokyo, Hongo, Bunkyo-ku, Tokyo 113-0033, Japan \\ 4 National Astronomical Observatory of Japan, National Institutes of Natural Sciences, Mitaka, Tokyo 181-8588, Japan \\ Received 16 November 2007 / Accepted 7 January 2008
}

\section{ABSTRACT}

\begin{abstract}
Aims. We study the physical properties of an active region (AR) jet in order to probe the mechanisms responsible for it. Methods. We report 2007 January 15/16 observations of a recurring jet situated on the west side of NOAA AR 10938. Multiwavelength data from all three instruments onboard Hinode were analysed. This paper focuses on one instance of a jet observed with the Hinode/EUV Imaging Spectrometer (EIS). Using EIS raster data we measured the temperatures, Doppler shifts, density, and filling factor.

Results. A strong blue-shifted component and an indication of a weak red-shifted component at the base of the jet was observed around $\log T_{\mathrm{e}}=6.2$. The up-flow velocities exceeded $150 \mathrm{~km} \mathrm{~s}^{-1}$. The jet component was seen over a range of temperatures between 5.4 and 6.4 in $\log T_{\mathrm{e}}$. Using Fe XII $\lambda 186$ and $\lambda 195$ line ratios, we measured densities above $\log N_{\mathrm{e}}=11$ for the high-velocity up-flow component. We found that the density of the high-velocity up-flow increases with velocity. We estimate the filling factor in the jet upflow to be $<0.03$. With the Hinode/Solar Optical Telescope (SOT), we observed recurrent (quasi periodic) magnetic flux cancelations just before the recurrent jet emission was seen in images taken with the X-ray Telescope (XRT).

Conclusions. The high-velocity up-flows, together with the density dependence on velocity, support an evaporation scenario for the acceleration of this jet. The high density and small filling factor, coupled with the high Doppler velocities are strongly suggestive of multiple small-scale magnetic reconnection events being responsible for the production of both EUV and X-ray jets.
\end{abstract}

Key words. Sun: activity - Sun: corona - Sun: flares - Sun: magnetic fields - Sun: - UV radiation - Sun: X-rays, gamma rays

\section{Introduction}

A variety of jet-like phenomena are observed in the solar atmosphere, including plasma ejections over a range of coronal temperatures, which one observes as extreme ultraviolet (EUV) and X-ray jets. X-ray jets were discovered with the Soft X-ray Telescope (SXT: Tsuneta et al. 1991) on board Hinode's predecessor, Yohkoh. They are defined as transient X-ray enhancements with apparent collimated motion and are associated with microflares, occurring in active regions (ARs) or X-ray bright points (Shimojo et al. 1996). Shimojo et al. (1998) show that most X-ray jets $(70 \%)$ are located in mixed magnetic polarity regions or satellite polarity regions. While it is strongly believed that X-ray jets are produced by magnetic reconnection, the processes at work during reconnection have not yet been fully understood. Moreover, one fundamental question remains: How are jets accelerated in the magnetic reconnection process? While it has been proposed by Shimojo et al. (1996) and Miyagoshi \& Yokoyama (2004) that jets are the evaporation flow produced by reconnection, only apparent velocities of X-ray jets have so far been measured with SXT to support this scenario. To date, the relationships between X-ray jets and cooler ejections (e.g. EUV jets) have not been well established.

The recent launch of the Hinode solar observatory (Kosugi et al. 2007) is a perfect opportunity to tackle these questions. With Hinode, we are able to obtain simultaneous multiwavelength observations of jets with high spatial and spectral resolution, as well as detailed magnetic field information. We present a study of an AR jet observed by all three instruments on board Hinode on 2007 Jan. 15/16 (west of NOAA AR 10938). In this Letter, we mainly focus on the observation of a jet instance for which we have EUV Imaging Spectrometer (EIS) coverage. A more comprehensive study including detailed observations from all Hinode instruments, with complementary data from RHESSI and Stereo/SECCHI/EUVI is in preparation (Chifor et al. 2008).

\section{Observations and data reduction}

We began our observations on 2007 Jan. 15 at $~ 22: 15$ UT when we observed a persistent X-ray brightening and recurrent jets west of the AR with the Hinode/X-ray Telescope (XRT: Golub et al. 2007) in its "Thin Al_poly" filter. The X-ray jets reoccurred at the same location several times between 22:16 and 02:12 UT (Fig. 1), with the largest jet taking place 22:28 UT.

Solar Optical Telescope (SOT: Tsuneta et al. 2007) images of this event revealed that the jets were located close to a pore, west of the AR, in a region of canceling magnetic flux. Figure 1 (top row) shows four instances of the XRT X-ray jets with simultaneous contours of the SOT FG/Stokes V images overlaid. The SOT images have been co-aligned using the offsets given by Shimizu et al. (2007), with a precision of $2^{\prime \prime}$. Starting with 01:54 UT on Jan. 16, the EUV Imaging Spectrometer (EIS: Culhane et al. 2007) onboard Hinode ran its observing study "HH_AR+FLR_RAS_N01(J)" three times over the AR. Around 

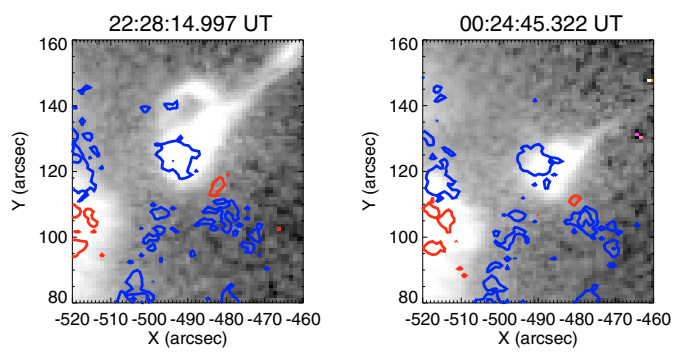

01:12:26.272 UT
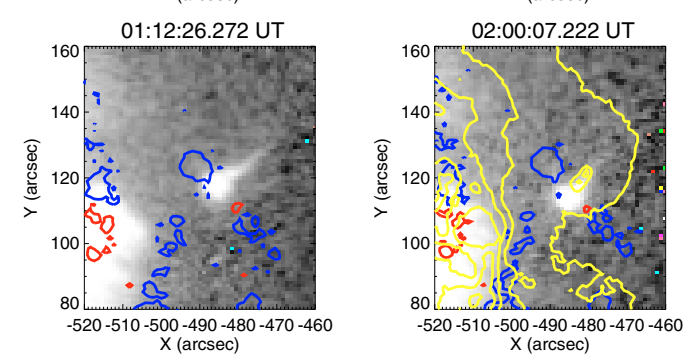

Fig. 1. Instances of the recurring X-ray jet observed by XRT between 22:28-02:00 UT, gradually decreasing in height and intensity. SOT/FG Stokes V contours are overplotted (positive polarity in blue and negative polarity in red). SOT images were shifted $x+37.2^{\prime \prime}$ and $y+23.5^{\prime \prime}$ (offsets from Shimizu et al. 2007). EIS Fe XII $\lambda 195$ contours are overplotted (in yellow) on the XRT image taken at 02:00 UT, the time when EIS rasters over the jet.

the time when EIS rastered over the X-ray jet area ( 02:00 UT), we co-aligned the EIS and XRT images using the raster taken by EIS in Fe XV $\lambda 284$. We estimate the errors associated with this co-alignment method to be less than $3^{\prime \prime}$. The EIS raster observed the last of the X-ray jets before the X-ray brightening disappeared completely. The bright emission detected by EIS in the Fe XII $\lambda 195$ line matches the location and direction of the $\mathrm{X}$-ray jets (Fig. 1, bottom right panel).

The Fe $6302 \AA$ Stokes I and V observations were obtained by SOT through the narrow-band filter (NFI). From the Stokes I and $\mathrm{V}$ images at $-120 \mathrm{~m} \AA$ of the Fe $6302 \AA$ line, we made preliminary calculations of the line-of-sight magnetic field in Gauss units as $B=-Q \times\left(C_{v} / 0.798\right) / C_{i}$, where $C_{v}$ and $C_{i}$ are the counts of Stokes $V$ and Stokes $I$ images, respectively, and $Q$ is a calibration coefficient (Chae et al. 2007). We observed a recurrent decrease in the magnetic flux (almost step-wise for the negative flux), just before the ejection of each X-ray jet. Measurements of the magnetic fluxes and energy release during this event are in progress. Their correlation with the jet formation will be reported by Chifor et al. (2008).

EIS covers two wavelength bands: 170-211 $\AA$ and 246-292 $\AA$, referred to as the short wavelength (SW) and long wavelength (LW) bands, respectively. The "HH_AR+ FLR_RAS_N01(J)" observing sequence uses the 1" slit to raster over an area of $240^{\prime \prime} \times 240^{\prime \prime}$ with 5-s exposure times, giving a total duration of $26 \mathrm{~min}$. We used one EIS AR raster taken between 01:54:11-02:20:27 UT. The EIS rastering took place over the location of the recurrent X-ray jet (seen in XRT images) between $~ 02: 00-02: 01 \mathrm{UT}$.

To obtain calibrated intensities in units of erg $\mathrm{cm}^{-2} \mathrm{~s}^{-1} \mathrm{sr}^{-1} \AA^{-1}$ at each pixel in the data set, we applied the standard processing routine eis_prep available in the Solarsoft tree. This routine removes the dark current and CCD pedestal, as well as hot pixels and cosmic ray hits. The spectrum at each pixel was then fitted with a Gaussian function using the eis_auto_fit routine. We shifted the LW images by $2^{\prime \prime}$
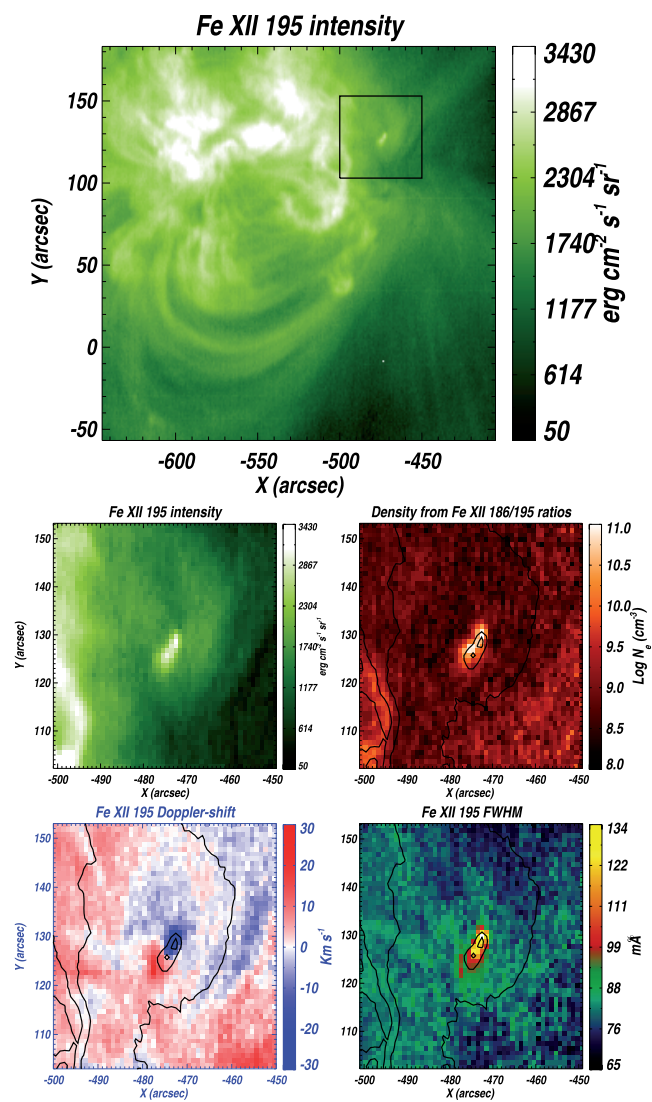

Fig. 2. Top panel: FeXII $\lambda 195$ intensity map obtained between 01:54-02:20 UT. EIS rasters over the jet feature between 02:00-02:01 UT. The FOVs of the middle and bottom panels are marked by the square. Middle panels: Fe XII $\lambda 195$ intensity map and density map obtained from the FeXII $(\lambda 186 / \lambda 195)$ line ratio diagnostics. Bottom panels: the Fe XII $\lambda 195$ Doppler-shift and line FWHM maps. A pair of blue/red-shifted components are conspicuous in the Doppler-shift map. Overlaid are the Fe XII $\lambda 195$ line intensity contours.

in the solar $X$ direction and $17^{\prime \prime}$ in the solar Y direction. This correction takes into account the instrumental offset between the images taken in the two EIS CCDs (Young et al. 2007b).

To extract Doppler-shift information, one needs to be aware that the EIS slit is not perfectly vertical on the CCD, and that there is an orbital variation of the spectral line position due to the thermal changes across the instrument. We obtained the slit tilt using the eis_wave_corr routine and then subtracted it from the fitted line centre positions. The eis_orbit_spline routine also available in the EIS branch of SolarSoft fits a spline to the variation of the lines within the raster and applies the orbital variation correction. Because the EIS raster images in our case are dominated by the AR (with flows present), a spline fit including all pixels in the raster would not remove the orbital variation correctly. We therefore used only the quieter region (with no significant dynamic activity) in the Fe XII raster (bottom rows of pixels $0-50$ ) to obtain the orbital variation. The result was then subtracted from the line centre positions measured in all wavelength windows.

Figure 2 shows the Fe XII $\lambda 195$ intensity, Doppler-shift, and line width maps. To compute the relative Doppler velocities, we assumed the Fe XII reference wavelength is equal to the average centre of lines fitted in the quiet part (bottom rows of pixels $0-50$ ) of the raster. We found a prominent blue-shifted component of the jet (roughly aligned $\mathrm{N}-\mathrm{W}$ in projection) and a 

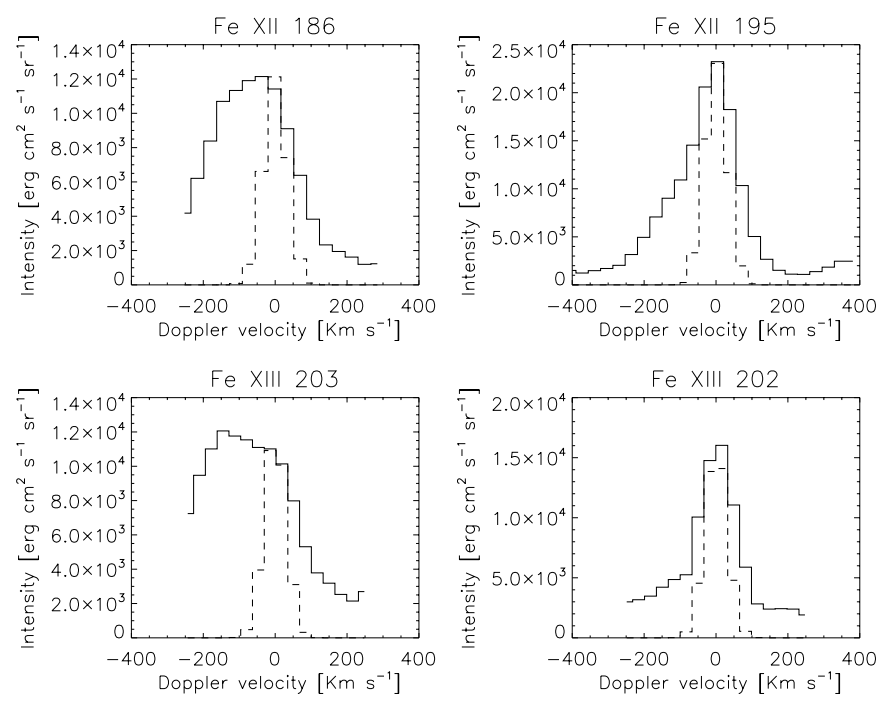

Fig. 3. Profiles of the density-sensitive line pairs from the blue-shifted part of the jet: Fe XII $\lambda 186, \lambda 195$ and Fe XIII $\lambda 203, \lambda 202$. Spectra were averaged over a $4 \times 4$ pixel region around the high-density, blue-shifted feature. Overplotted (dotted lines) are reference line profiles obtained using a FWHM of $0.05 \AA$. The zero-velocity wavelengths were determined relative to the Fe XII reference wavelength.

weaker red-shifted component (at its S-E "base"). The jet was observed at temperatures between 5.4 and 6.4 in $\log T_{\mathrm{e}}$. In the Ca XVII $\lambda 192$ window at the location of the up-flow jet component, the OV $\lambda 192.90$ line was seen next to the $\lambda 192.83$ line (believed to be Fe XI, Young et al. 2007a). We note that there is also $\mathrm{OV}$ emission near the base (red-shifted component) of the jet, but this could be due to the red-shifted Fe XI line. We also observed the jet in the Fe VIII, Si VII, Fe X, Fe XII, Fe XIII, and Fe XV lines. The Fe XVI emission was observed at the base.

Profiles of density diagnostic line pairs at temperatures between $\log T_{\mathrm{e}}=6.2-6.3$ are shown in Fig. 3. Spectra were averaged over a $4 \times 4$ pixel region around the high-density, blueshifted jet component. The blue-shift line wing components in Fig. 3 indicate high velocities. For example, Fe XII (ג195) and Fe XIII ( 1202) have blue-shifts greater than $150 \mathrm{~km} \mathrm{~s}^{-1}$ if we assume that there are two components present. Both Fe XII ( $\lambda 186)$ and Fe XII ( $\lambda 203)$ have blue-shift wings exceeding $175 \mathrm{~km} \mathrm{~s}^{-1}$. Furthermore, because the jet appears to be largely tilted from the line of sight, higher velocities are expected.

To determine the electron density of the jet, we used theoretical line intensity ratios of the Fe XII $(\lambda 186 / \lambda 195)$ densitysensitive pair. We calculate these ratios with the CHIANTI atomic package (Dere et al. 1997; Landi et al. 2006). The Fe XII $(\lambda 186 / \lambda 195)$ pair gives the best diagnostic due to its broad range of sensitivity $\left(\log N_{\mathrm{e}}=8-12\right.$, Young et al. 2007a). Both lines have self blends (at 186.85, 186.89, and 195.12, $195.18 \AA$, respectively), which we accounted for in our calculations. We have assessed the contribution of the S XI $\lambda 186.84$ line to the Fe XII $\lambda 186.88$ feature in AR conditions by using the unblended S XI line at $\lambda 188.68 \AA$ (not observed with the present study). The contribution is found to be at most $5 \%$, and typically $<3 \%$ in high-density plasmas $\left(\geq 10^{10} \mathrm{~cm}^{-3}\right)$, as the Fe XII lines are more sensitive to high densities than the S XI lines. The S XI blend has thus been ignored in the present work.

The density-dependent lines Fe XII $\lambda 186$ and Fe XIII $\lambda 203$ shown in Fig. 3 are particularly broad and blue-shifted. Their profiles are distinctly non-Gaussian and partially missing in the blue side due to the narrow window size (Fig. 3). Therefore,

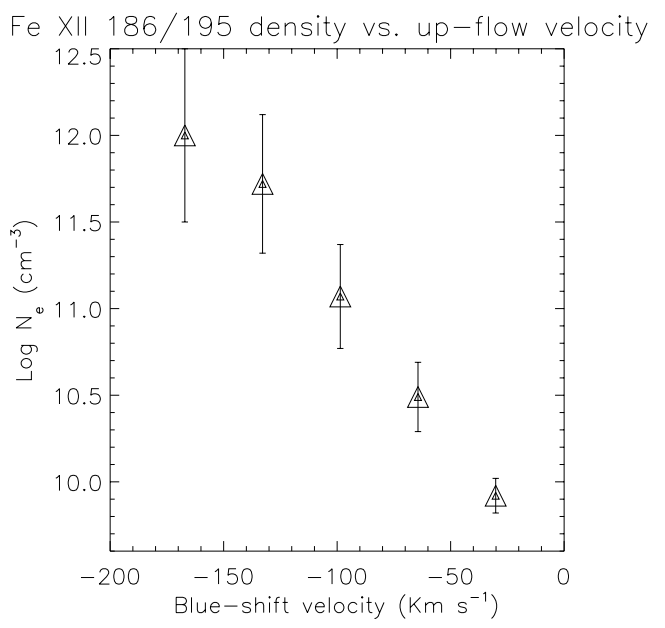

Fig. 4. Density values calculated from the Fe XII $(\lambda 186 / \lambda 195)$ profile ratio in the blue-shifted wings of the lines observed in the up-flow component. Density is increasing with the up-flow velocity.

instead of fitting these lines with one or more Gaussian profiles to obtain the density map in Fig. 2, we summed up the intensities in the wavelength windows $\lambda 186$ and $\lambda 195$. Figure 2 (middle right) shows the density map of the jet region. Using this method, the densities of the up-flow jet component were calculated to be $\log N_{\mathrm{e}}=11$. We note that an Fe XII line formed at $203.728 \AA$ is at most 0.25 of the 195 line (assuming a density of $N_{\mathrm{e}}=12$, with a lower percentage expected at $N_{\mathrm{e}}=11$ ). Therefore, the contribution of the Fe XII $203.728 \AA$ line blend to Fe XIII $\lambda 203$ does not explain its blue wing, with the most of the blue shift coming from the Fe XIII line itself.

When obtaining a density value in each pixel, we could not account for background emission. We thus employed a second method that accounts for background and calculates the density of the jet as a function of up-flow velocity. From both Fe XII profiles shown in Fig. 3, we subtracted a linear background emission estimated from the Fe XII $\lambda 195$ window to be $10^{3} \pm 500 \mathrm{erg} \mathrm{cm}^{2} \mathrm{~s}^{-1} \mathrm{sr}^{-1} \AA^{-1}$. Within the given uncertainty, this value is consistent with the background level estimated over the entire LW range. We then divided the background-subtracted $\lambda 186$ and $\lambda 195$ line profiles shown in Fig. 3, on the blue-shifted side of their spectra. Figure 4 shows that, in the observed jet, density increases with the up-flow velocity. We estimated the errors in the line intensities and those due to background subtraction intensity and used the CHIANTI dens_plotter routine to calculate the errors in the densities shown in Fig. 4. We note that the Fe XII $\lambda 186$ self blend produces a velocity difference of $49 \mathrm{~km} \mathrm{~s}^{-1}$, which is significantly less than the upflow magnitude around $200 \mathrm{~km} \mathrm{~s}^{-1}$. Furthermore, the $186.85 \AA$ line is only about half as strong as the $186.88 \AA$ line. Therefore, the conclusion that the high velocities have the highest density is not affected by the self blend.

Using the derived density, one can estimate the plasma emission column depth by assuming an isothermal plasma at $\log T_{\mathrm{e}}=$ 6.1 and a coronal abundance for iron $(\log [\mathrm{Fe} / \mathrm{H}]=8.10$, Feldman et al. 1992). The high density coupled with a modest intensity leads to a column depth of around $50 \mathrm{~km}$. The extent of the jet in the plane of the sky is around 2-3" (Fig. 3), making the filling factor of the jet around 0.03 . This suggests that the jet consists of multiple small-scale, high-density structures. Coupled with the high velocities, these observations are strongly 
suggestive of multiple small-scale magnetic reconnection events taking place within the jet.

\section{Conclusions and future work}

In this Letter, we have reported the analysis of an AR jet observed with all instruments onboard Hinode, focusing mainly on the observations taken with EIS. We found plasma emission during the jet co-existing over a range of temperatures $\left(\log T_{\mathrm{e}}=\right.$ 5.4-6.4). A strong blue shift with up-flows velocities exceeding $150 \mathrm{~km} \mathrm{~s}^{-1}$ was observed, as well as an an indication of a weak red-shift component at the jet footpoint. We note that, based on the SOT magnetic field configuration and the XRT data, one can be confident that EIS indeed observed a jet instance, rather than a flow in a loop with a red shift in one footpoint and a blue shift at the other. We measured densities above $\log N_{\mathrm{e}}=11$ for the high-velocity up-flow component, from which we derived a small filling factor $(<0.03)$. The high densities and small filling factor indicate that the energy deposition occurred only in selected field lines, which may be related to the fine structure observed in many flaring events.

The high jet density is indicative of emission coming from the chromosphere in the process of evaporation. Shimojo et al. (1996) made a statistical study of X-ray jets from those observed by SXT, measuring their apparent velocities between $10-10^{3} \mathrm{~km} \mathrm{~s}^{-1}$, with an average of $200 \mathrm{~km} \mathrm{~s}^{-1}$. Our calculated Doppler velocities represent the line-of-sight component of X-ray jets, close in value to the apparent velocities and to values expected from a chromospheric evaporation flow.

The correlation between the up-flow velocity and density supports the evaporation scenario. Shimojo et al. (2001) performed a one-dimensional hydrodynamic simulation of a jet, showing that both velocity and density correlate with the heating rate in the corona. During evaporation, the jet is driven by the gas pressure gradient. The model predicts the higher velocities and densities associated with a higher pressure gradient.

Similar to the analysis of a polar jet by Kamio et al. (2007), we found indications of a red-shifted footpoint of the AR jet, which is not consistent with the evaporation scenario. If one assumes that the up-flow and down-flow both represent a bipolar reconnection jet (directly accelerated by the $J \times B$ force of the reconnected field lines), one would expect an anti-correlation of velocity and density. This is because the velocity of a reconnection jet is determined by the Alfvén velocity, which decreases with density. A more detailed analysis of the EIS data, including line wavelength calibrations and blends is required.

The magnetic topology that precedes an evaporation jet from a cancelation event is not obvious, so we will investigate it by careful co-alignment of XRT and SOT data. Chifor et al. (2008, in preparation) will present a more detailed analysis of the series of jets we observed on 2007 Jan. 15/16, including RHESSI and Stereo/SECCHI/EUVI complementary data.

Acknowledgements. Hinode is a Japanese mission developed and launched by ISAS/JAXA, with NAOJ as domestic partner and NASA and STFC (UK) as international partners. It is operated by these agencies in co-operation with ESA and NSC (Norway). We thank G. Del Zanna, K. Ichimoto, Y. Katsukawa, and T. Shimizu for their help. CC is supported by the University of Cambridge Trusts and an Isaac Newton Studentship. H.E.M. and D.T. acknowledge support from STFC.

\section{References}

Chae, J., Moon, J.-Y., Park, Y.-D., et al. 2007, PASJ, 59, 619

Chifor, C., Isobe, H., Mason, H. E., et al. 2008, A\&A, in preparation

Culhane, J. L., Harra, L. K., James, A. M., et al. 2007, Sol. Phys., 243, 19

Dere, K. P., Landi, E., Mason, H. E., Monsignori Fossi, B. C., \& Young, P. R. 1997, A\&AS, 125, 149

Feldman, U., Laming, J. M., Mandelbaum, P., Goldstein, W. H., \& Osterheld, A. 1992, ApJ, 398, 692

Golub, L., Austin, G., Bookbinder, J., et al. 2007, Sol. Phys., 243, 63

Isobe, H., Tripathi, D., \& Archontis, V. 2007, ApJ, 657, L53

Kamio, S., Hara, H., Watanabe, T., et al. 2007, PASJ, 59, 757

Kosugi, T., Matsuzaki, K., Sakao, T., et al. 2007, Sol. Phys., 243, 3

Landi, E., Del Zanna, G., Young, P. R., et al. 2006, ApJS, 162, 261

Miyagoshi, T., \& Yokoyama, T. 2004, ApJ, 614, 1042

Shimizu, T., Katsukawa, Y., Matsuzaki, K., et al. 2007, PASJ, 59, 845

Shimojo, M., Hashimoto, S., Shibata, K., et al. 1996, PASJ, 48, 123

Shimojo, M., Shibata, K., \& Harvey, K. L. 1998, Sol. Phys., 178, 379

Shimojo, M., Shibata, K., Yokoyama, T., \& Hori, K. 2001, ApJ, 550, 1051

Tsuneta, S., Acton, L., Bruner, M., et al. 1991, Sol. Phys., 136, 37

Tsuneta, S., Ichimoto, K., Katsukawa, Y., et al. 2007, Sol. Phys., submitted [arXiv: 0711.1715v1]

Yokoyama, T., \& Shibata, K. 1996, PASJ, 48, 353

Young, P. R., Del Zanna, G., Mason, H. E., et al. 2007a, PASJ, 59, 857

Young, P. R., Del Zanna, G., Mason, H. E., et al. 2007b, PASJ, 59, 727 